\title{
Determination and optimization of mode matching into optical cavities by heterodyne detection
}

\author{
Guido Mueller, Qi-ze Shu, ${ }^{*}$ Rana Adhikari, ${ }^{\dagger}$ D. B. Tanner, and David Reitze \\ Department of Physics, University of Florida, Gainesville, Florida 32611-8440 \\ Daniel Sigg \\ LIGO Hanford Observatory, P.O. Box 1970 S9-02, Richland, Washington 99352 \\ Nergis Mavalvala and Jordan Camp \\ LIGO Project, California Institute of Technology, MS 18-34, Pasadena, California 91125
}

Received November 4, 1999

\begin{abstract}
We report on a novel high-sensitivity method to characterize and improve mode matching into optical cavities. This method is based on heterodyne detection of cylindrical transverse cavity modes. A specially designed annular-segmented photodiode is used to measure the amplitude of nonresonant modes reflected by the cavity. Our measurements allow us to optimize cavity mode matching to nearly $99.98 \%$ and will play an important diagnostic role in gravitational-wave detectors. (c) 2000 Optical Society of America

OCIS codes: $\quad 040.2840,120.0120,120.2230,140.4780$.
\end{abstract}

Heterodyne methods have become well established for sensing and controlling optical cavities with extremely high precision. These methods include the PoundDrever-Hall scheme ${ }^{1}$ for controlling cavity lengths (matching longitudinal cavity and laser modes) and its extension by Anderson ${ }^{2}$ and Sampas and Anderson ${ }^{3}$ for sensing and controlling cavity alignment. The former method is universally used for cavity locking; the latter method was recently developed for detection of off-axis cavity modes and autoalignment of cavities. ${ }^{3-6}$

Both methods play a key role in the development of gravitational-wave (GW) interferometers. Several large-scale interferometers are currently under construction worldwide with the goal of detecting gravitational waves from astrophysical sources. ${ }^{7,8}$ To enhance GW detection sensitivity, many GW projects have adopted a design in which a Fabry-Perot cavity is placed in each arm of a Michelson interferometer. The shot-noise-limited sensitivity of these interferometers depends on the precision with which the length stabilization can be maintained, the degree of cavity alignment, and the total power that can be efficiently coupled into the arm cavities. This power, in turn, depends on the degree of mode matching of the transverse modes into the resonant cavities.

Measurements of cavity alignment utilize the fact that higher-order modes are introduced when the mode parameters of the input beams do not match the cavity eigenmodes. $^{2}$ If an input beam is slightly misaligned in angle or transversely displaced with respect to the cavity axis, first-order Hermite-Gauss modes are introduced. Sampas and Anderson ${ }^{3}$ and Hefetz et al. ${ }^{4}$ have demonstrated that, by using phase modulation sidebands and quadrant photodetectors, one can detect and control cavity misalignments by measuring the transmitted $^{3}$ or reflected ${ }^{4}$ cavity light. Similarly, if the beam waist's size and position do not match the cavity waist's size and position, second-order HermiteGauss modes are introduced.

0146-9592/00/040266-03\$15.00/0
In this Letter we present, for the first time to our knowledge, a method for accurately measuring and correcting for mode mismatch by heterodyne detection of second-order Hermite-Gauss modes. Our method has several advantages over other techniques. Because it uses heterodyne detection, it provides an error signal that can be used for feedback to adaptive optical components. In addition, it directly provides information related to the waist position and size differentials between the input mode and the cavity. Unlike cavity scanning techniques, it can be used with complex, coupled cavities, as for example in most GW detectors. Finally, it is nonperturbative and can be used for dynamic in situ measurements without disturbing the cavity. The last-named advantage is particularly important for high-power applications in which dynamic (power-dependent) thermal lensing caused by bulk and surface deformations in optical components alters the mode matching. ${ }^{9}$ For the interferometric detectors that make up the Laser Interferometer Gravitational-Wave Observatory (LIGO) at Hanford, Wash., and Livingston, La., thermal lensing is not inconsequential: As much as $300 \mathrm{~W}$ of resonant power is transmitted through and $10 \mathrm{~kW}$ is reflected from selected optical components of the interferometer, resulting in thermal lenses that change the mirrors' effective radii of curvature by as much as $30 \%{ }^{10}$ Accurately quantifying these changes is essential for optimizing the performance of interferometric GW detectors.

The setup of our measurement technique is shown in Fig. 1. We use a Nd:YAG nonplanar ring oscillator, which produces a single longitudinal mode. The laser field is spatially filtered by a mode cleaner ${ }^{11}$ (not shown in Fig. 1). The transverse mode of the field transmitted through the mode cleaner is a fundamental Hermite-Gaussian mode with one longitudinal frequency. The phase modulator (EOM) superimposes sidebands onto this field. The laser frequency is 


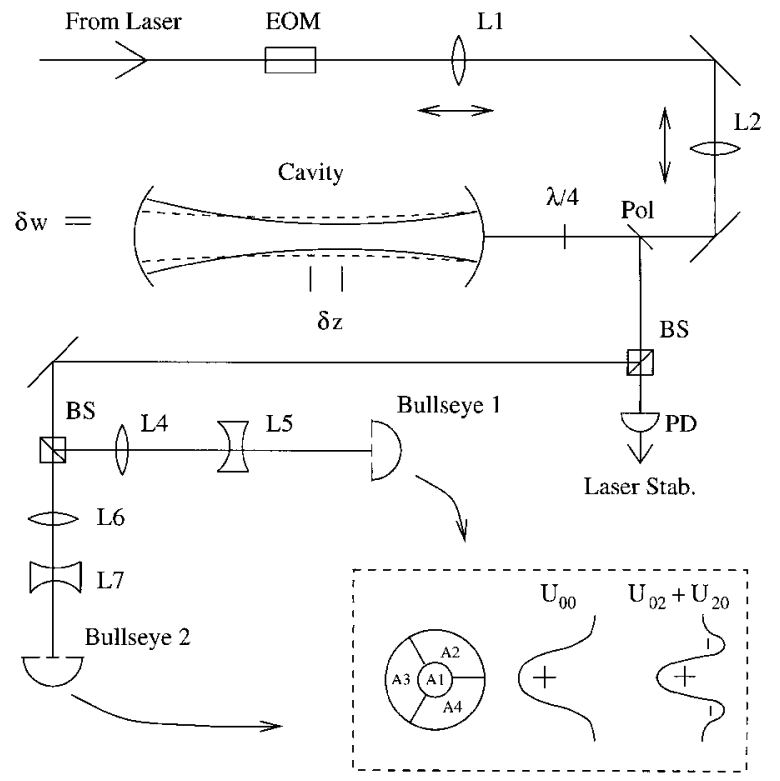

Fig. 1. Experimental setup for measuring mode matching showing the incoming field, the EOM, lenses L1 and L2 for mode matching, wave plate $\lambda / 4$, polarizer Pol, the cavity, two beam splitters BS, photodiode PD for length control, and the two BPD's. The Gouy-phase telescopes (L4, L5) and (L6, L7) match the Gouy phases and the size of the beams to the detectors. Inset, geometry of the BPD's and the fundamental and higher-order field eigenmodes. Stab., signal used to stabilize the frequency of the laser onto the eigenfrequency of the cavity.

locked to a longitudinal resonance of the cavity. The transverse mode is relayed to the cavity through lenses L1 and L2. By moving the lenses we can adjust the mode matching into the cavity. The cavity has a free spectral range of $850 \mathrm{MHz}$ with mirrors that have a nominal transmission of 100 parts in $10^{6}$ (corresponding to a finesse of 30,000). The first cavity mirror is flat, and the second mirror has a radius of curvature of $1 \mathrm{~m}$. By using annular segmented photodiodes [bulls-eye photodetectors (BPD's)] and a modulation-demodulation technique, we extract a signal proportional to the degree of mode mismatch by detecting the beat between the higher-order modes of the input field with the fundamental mode of a local oscillator and vice versa. Each of the BPD's used in the experiment has four active areas, as shown in the inset of Fig. 1. This design is superior to a design with two active ring areas because a linear combinations of the signals of the outer segments can be used to align the input beam relative to the cavity ${ }^{5}$ and to center the beam on the BPD. To adjust the phase difference between the fundamental and second-order Hermite-Gauss modes caused by the Gouy phase shift, we use lenses L4 and L5 and lenses L6 and L7 as Gouy-phase telescopes ${ }^{12}$ for the BPD's.

Mode mismatch can be quantified with two parameters: the difference in waist position $\delta z$ of the input field relative to the waist position $\left(\hat{z}_{0}\right)$ of the cavity eigenmodes and the difference between beam size $w$ of the input field at $\hat{z}_{0}$ and the waist $\hat{w}$ of the eigenmodes of the cavity, $\delta w=\hat{w}-w$ (see the cavity in Fig. 1). For small mismatches, the laser field mode $\left(U_{00}\right)$ expressed in the eigenmodes of the cavity $\left(\hat{U}_{n m}\right)$ at the waist of the cavity is ${ }^{2}$

$$
\begin{aligned}
U_{00} & =\hat{U}_{00}-b\left(\hat{U}_{20}+\hat{U}_{02}\right), \\
b & =\left(\frac{\delta w}{\sqrt{2} \hat{w}}+i \frac{\delta z}{\sqrt{2} \hat{z}_{R}}\right),
\end{aligned}
$$

where $\hat{z}_{R}$ is the Rayleigh range of the cavity eigenmodes and an overall phase is ignored. The component at frequency $\Omega$ of the difference between the central segment photocurrent and the outer segment photocurrent of each BPD is

$$
\begin{aligned}
S \propto & \sin (\Omega t) \operatorname{Im}\left\{r_{\mathrm{FP}}\left[\int_{A 1}\left|\hat{U}_{00}\right|^{2}-\int_{A 0}\left|\hat{U}_{00}\right|^{2}\right]\right. \\
& +b^{*}\left(1-r_{\mathrm{FP}}\right)\left[\int_{A 1} \hat{U}_{00}\left(\hat{U}_{20}{ }^{*}+\hat{U}_{02}{ }^{*}\right)\right. \\
& \left.\left.-\int_{A 0} \hat{U}_{00}\left(\hat{U}_{20}{ }^{*}+\hat{U}_{02}{ }^{*}\right)\right]\right\},
\end{aligned}
$$

where $\Omega$ is the $\mathrm{rf}$ applied to the EOM, $r_{\mathrm{FP}}$ is the amplitude reflection coefficient of the cavity for the carrier in the fundamental cavity mode, and $A 1$ and $A 0=(A 2+A 3+A 4)$ are the areas of circular central and the annular outer segments, respectively, of the BPD's. $\quad r_{\mathrm{FP}}$ is real if the input field is perfectly locked to a longitudinal eigenmode of the cavity. It is zero for a perfectly impedance matched cavity, positive for an undercoupled cavity, and negative for an overcoupled cavity. The result of the first integration in relation (3) is a real number that depends only on the physical dimensions of the BPD's. The result of the second integration is a complex number. Its amplitude depends also on the dimensions of the BPD's, but its phase is equal to the phase difference of the two modes and twice the Gouy phase picked up between the cavity and the BPD. Each BPD is preceded by Gouy-phase telescopes. These telescopes increase the beam size in such a way that the intensity of the $\hat{U}_{00}$ mode impinging upon the central areas (A1) of the BPD's is equal to the intensity of the same mode impinging upon the corresponding outer area $(A 0): \quad\left(\int_{A 1}\left|\hat{U}_{00}\right|^{2}-\int_{A 0}\left|\hat{U}_{00}\right|^{2}\right)=0$. Note that $\left(\hat{U}_{02}+\hat{U}_{20}\right)$ changes sign near the border between $A 1$ and $A 0$. The telescopes also adjust the phase difference to be $180^{\circ}$ for the first $\mathrm{BPD}$ and $270^{\circ}$ for the second one. Therefore the integral gives a pure real value at the first BPD detector and a pure imaginary value at the second one.

The BPD photocurrents are demodulated with a mixer. For the appropriate Gouy phase, the demodulated signal $D_{1}$ is proportional to the mismatch in the waist position and $D_{2}$ is proportional to the difference in waist size:

$$
\begin{aligned}
& D_{1}=A_{1}\left(1-r_{\mathrm{FP}}\right) \operatorname{Im}(b) \propto \delta z, \\
& D_{2}=A_{2}\left(1-r_{\mathrm{FP}}\right) \operatorname{Re}(b) \propto \delta w,
\end{aligned}
$$



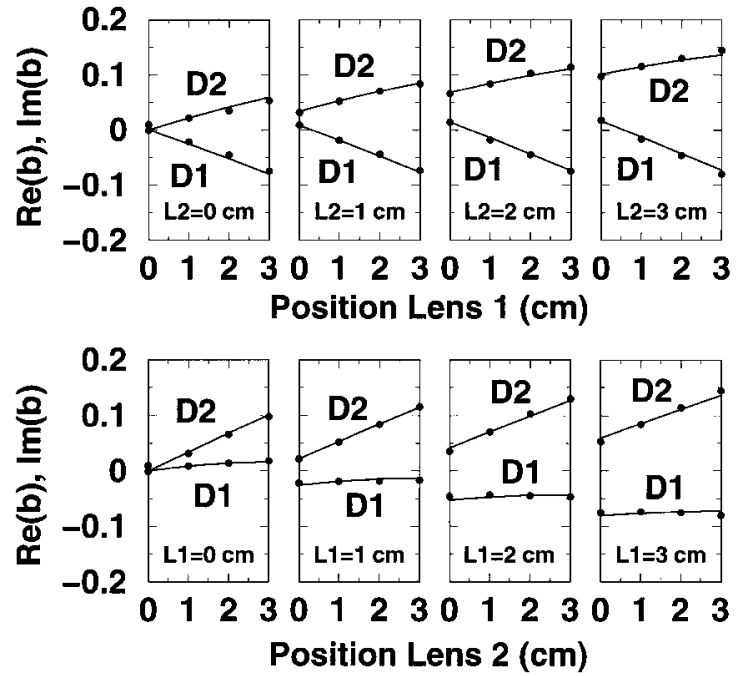

Fig. 2. Experimental (filled circles) and theoretical (lines) demodulated signals for various combinations of lens positions. The demodulated signals are scaled to be proportional to the real part (D2) and the imaginary part (D1) of the amplitude $b$ of the laser field in the second-order Hermite-Gaussian eigenmode of the cavity.

where $A_{1}$ and $A_{2}$ are scaling factors and include the modulation index, the intensity of the input field, the transmissivities and reflectivities of optical elements between the BPD's and the cavity, the efficiency of the active areas of the detectors, the electronic gains, and the mixer efficiencies.

We measured the BPD signals for various lens positions and compared them with the expected values. The results can be seen in Fig. 2, where we plot the demodulated BPD signals for various mode mismatches created by moving lenses L1 and L2. The top of the figure displays both demodulated signals for the case when the position of $\mathrm{L} 1$ varies and L2 is fixed at 0 , 1,2 , and $3 \mathrm{~cm}$. In the bottom of the figure L2 varies and $\mathrm{L} 1$ is fixed at $0,1,2$, and $3 \mathrm{~cm}$. The points are the measured values and the solid lines are the theoretically expected values for the mode matching based on Gaussian modal theory. ${ }^{13}$ The numerical results are scaled such that for small mismatches D1 is equal to $\delta z / 2 \sqrt{2} \hat{z}_{R}$ and D2 is equal to $\delta w / \sqrt{2} \hat{w}$. The experimental values are scaled with only one fitting parameter for each detector. The agreement between theoretical and experimental results is quite good. The theory reproduces all offsets and slopes. At best matching, the amplitude of the second-order HermiteGauss mode dropped to $<1.5 \times 10^{-2}$, resulting in a lower limit of mode matching of $99.98 \%$.

The reflected power dropped at the optimum point to $2.6 \%$ of the impinging power. Inasmuch as the phasemodulation index is $0.16,1.3 \%$ of the total incident power is in these phase-modulation sidebands, which reflect from the cavity. Thus almost $99 \%$ of the incident power is transmitted through (or absorbed in) this high-finesse cavity. The remaining $1.3 \%$ reflection could be caused by higher-order modes in the input field, which cannot be mode matched, or, most probably, by impedance mismatching.
The results of our experiment demonstrate that mode mismatch can be accurately measured with a phase-modulation technique. This method has the advantage of giving signals that are linear in waist position and waist size deviations. It is accurate in the presence of other imperfections, such as misalignment of optical components, beam offsets, and optical losses, and delivers in situ information about the mode matching that is important for monitoring the thermal lensing in various optical components.

This technique is being implemented as an important diagnostic tool for the LIGO detectors and is currently tested ${ }^{14}$ or proposed ${ }^{15}$ for other GW detectors. We also foresee applications of the technique as a diagnostic tool in areas that deal with thermal lensing and adaptive optics.

This research was supported by National Science Foundation grants PHY-9722114 and PHY-9210038. We gratefully acknowledge helpful comments from Stan Whitcomb, David Shoemaker, and Albert Lazzarini. G. Mueller's e-mail address is mueller@ phys.ufl.edu.

*Present address, LOTS Technology, Inc., Sunnyvale, California 94089-1122.

${ }^{\dagger}$ Present address, LIGO Project, Massachusetts Institute of Technology, Cambridge, Massachusetts 02139 .

\section{References}

1. R. W. P. Drever, J. L. Hall, F. V. Kowalski, J. Hough, G. M. Ford, A. J. Munley, and H. Ward, Appl. Phys. B 31, 97 (1983).

2. D. Z. Anderson, Appl. Opt. 23, 2944 (1984).

3. N. M. Sampas and D. Z. Anderson, Appl. Opt. 29, 394 (1990).

4. Y. Hefetz, N. Mavalvala, and D. Sigg, J. Opt. Soc. Am. B 14, 1597 (1997).

5. N. Mavalvala, D. Sigg, and D. Shoemaker, Appl. Opt. 37, 7743 (1998).

6. E. Morrison, B. J. Meers, D. I. Robertson, and H. Ward, Appl. Opt. 33, 5041 (1994).

7. A. Abramovici and the LIGO group, Science 256, 325 (1992).

8. B. Caron and the VIRGO group, Class. Quantum Gravity 14, 1461 (1997).

9. W. Winkler, K. Danzmann, A. Ruediger, and R. Schilling, Phys. Rev. A 44, 7022 (1991).

10. D. E. McClelland, J. B. Camp, J. Mason, W. Kells, and S. E. Whitcomb, Opt. Lett. 24, 1014 (1999).

11. B. Willke, N. Uehara, E. K. Gustafson, R. L. Byer, P. J. King, S. U. Steel, and R. L. Savage, Opt. Lett. 23, 1704 (1998).

12. N. Mavalvala, "Alignment issues in Laser Interferometric Gravitational-Wave detectors," Ph.D. dissertation (Massachusetts Institute of Technology, Cambridge, Massachusetts, 1997).

13. A. Siegman, Lasers (University Science, Sausalito, Calif., 1986).

14. D. Shaddock, Department of Physics, Australian National University, Acton ACT 0200, Australia (personal communication, 1999).

15. B. Willke, Institut für Atom- und Molekühlphysik, Callinstrasse 38, D-30167 Hannover, Germany (personal communication, 1999). 